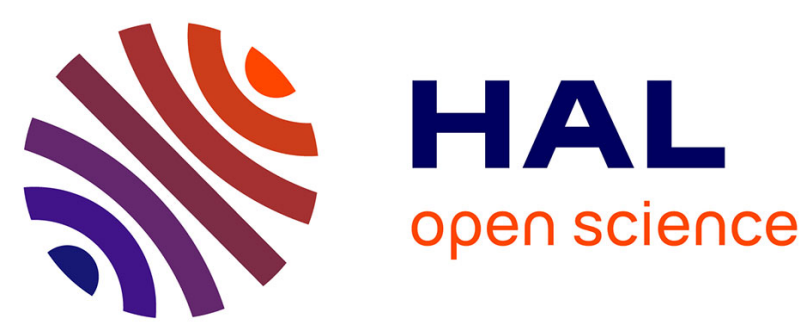

\title{
C-BORD - an overview of efficient toolbox for high-volume freight inspection
}

Pawel Sibczynski, A. Dziedzic, K. Grodzicki, J. Iwanowska-Hanke, Z.

Mianowska, M. Moszynski, L. Swiderski, A. Syntfeld-Kazuch, M. Szawlowski, D. Wolski, et al.

\section{To cite this version:}

Pawel Sibczynski, A. Dziedzic, K. Grodzicki, J. Iwanowska-Hanke, Z. Mianowska, et al.. C-BORD - an overview of efficient toolbox for high-volume freight inspection. 2017 IEEE Nuclear Science Symposium and Medical Imaging Conference (NSS/MIC), Oct 2017, Atlanta, United States. pp.1-3, 10.1109/NSSMIC.2017.8532735 . hal-01990519

\section{HAL Id: hal-01990519 https://hal.science/hal-01990519}

Submitted on 24 Jan 2019

HAL is a multi-disciplinary open access archive for the deposit and dissemination of scientific research documents, whether they are published or not. The documents may come from teaching and research institutions in France or abroad, or from public or private research centers.
L'archive ouverte pluridisciplinaire HAL, est destinée au dépôt et à la diffusion de documents scientifiques de niveau recherche, publiés ou non, émanant des établissements d'enseignement et de recherche français ou étrangers, des laboratoires publics ou privés. 


\title{
C-BORD - an overview of efficient toolbox for high-volume freight inspection
}

\author{
Pawel Sibczynski, Andrzej Dziedzic, Krystian Grodzicki, Joanna Iwanowska-Hanke, Zuzanna Mianowska, \\ Marek Moszyński, Lukasz Swiderski, Agnieszka Syntfeld-Każuch, Marek Szawłowski, Dariusz Wolski, \\ Aleksandra Dołębska, Wojciech Gęsikowski, Jerzy Godlewski, Frédérick Carrel, Amélie Grabowski, Frederic Laine, \\ Guillaume Sannie, Adrien Sari, Sandra Moretto, Cristiano Fontana, Felix Pino, Bertrand Perot, Alix Sardet, Cedric \\ Carasco and beneficiaries of the C-BORD Consortium
}

\begin{abstract}
In the frame of the C-BORD project, five innovate technology pillars for Non-Intrusive Inspection (NII) are under development. Freight containers are potential means for smuggling, drug trafficking and transport of dangerous or illicit substances. The goal of the C-BORD project is to increase interdiction of illicit or dangerous materials in containerized freight and deliver new capabilities against critical operational requirements and constrains. Particularly, the aim of the project is to increase throughput of the container per time unit, reduce cost and tome of cargo inspection and minimize the false negative and false positive alarm ratios. Finally, thanks to field trials organized during the project, capability of these systems will be proven and the C-BORD Toolbox usefulness will be validated by end users under real conditions at sea and border crosses.
\end{abstract}

\section{INTRODUCTION}

$\mathrm{D}$ ETECTION of various threats or illicit materials on country borders plays important role in Homeland Security over the world. Every year the amount of transported goods is increasing, thus, effective and efficient solutions for cargo containers inspection are required in a day-to-day operations by Custom Offices. The higher throughput, increased sensitivity, improved image quality are highly demanded by end-users in order to detect possible illegal goods and, finally, execute a seizure. The non-intrusive inspection (NII) using new or upgraded technology would avoid the cargo physical examination, resulting in time lost and cost increase.

Manuscript received November 10, 2017. This project has received funding from the European Union's Horizon 2020 research and innovation programme under grant agreement No 653323. Disclaimer: This text reflects only the author's views and the Commission is not liable for any use that may be made of the information contained therein.

P. Sibczynski, A. Dziedzic, K. Grodzicki, J. Iwanowska-Hanke, Z. Mianowska, M. Moszyński, L. Swiderski, A. Syntfeld-Każuch, M. Szawłowski, D. Wolski are with the National Centre for Nuclear Research (NCBJ), Otwock, 05-400 Poland (telephone: +48 22273 1440, e-mail: pawel.sibczynski@ncbj.gov.pl, pawel.sibczynski@gmail.com)

A. Dołębska, W. Gęsikowski, J. Godlewski are with the Custom Office Gdynia, ul. Trzy Lipy 3, Gdańsk, Poland

F. Carrel, A. Grabowski, F. Laine, G. Sannie, A. Sari are with the CEA LIST, F-91191 Gif-Sur-Yvette, France

S. Moretto, C. Fontana, F. Pino are with the Istituto Nazionale di Fisica Nucleare, Via Marzolo 8, 35131 Padova, Italy

B. Perot, A. Sardet, C. Carasco are with the CEA, DEN, Cadarache, Nuclear Measurement Laboratory, F-13108 Saint-Paul-lez-Durance, France
Unfortunately, the NII used today cannot cope with all targets under all circumstances with equivalent efficiency.

The whole system, as a complete toolbox, would be able to efficiently detect the illegal and dangerous goods, which Custom Offices can face with, such as:

- Tobacco, alcohol, drugs, chemicals

- Explosives, weapons

- Radioactive and nuclear materials.

In the frame of the project not only the devices are developed, but also end-users requirements will be taken into account and tests in real conditions will be performed. The systems will be tested in three locations, where local Custom Offices requirements differ from each other:

- Large seaport - Rotterdam - stationary solutions

- Medium seaport - Deepwater Container Terminal (DCT) Gdańsk - relocatable solutions

- Land Border - Hungary - mobile solutions.

In this paper, we would like to provide general overview of the devices developed in the frame of C-BORD project [1] , supported by EU Horizon 2020 program.

\section{TECHNOLOGIES DEVELOPED IN THE FRAME OF C-BORD PROJECT}

\section{A. Radiation Portal Monitors}

The first technology is based on advanced Radiation Portal Monitors (RPM), designed and commercially available from Symetrica LTD [2]. The detectors, based on organic scintillators, will be used for gamma ray detection. Specialized software is used for classifying and identifying the radiation source. High Speed Naturally Occurring Radioactive Materials (NORM) management was implemented to the RPM systems, which can be provided as a fixed, relocatable or mobile solution.

\section{B. X-ray system}

The Smiths Detection HCVM-T ViZual 6032 towable X-ray system, which is a commercially available device [3], will be upgraded in the frame of C-BORD project. The HCVM-T trailer is equipped with dual energy linear accelerator, currently offering the throughput of up to 25 trucks per hour in scan mode and up to 150 trucks per hour in pass through mode. Main improvement will cover the classification of 
organic materials as well as global image quality. Moreover, the modernization will also cover the stationary solution, working currently at Rotterdam test site.

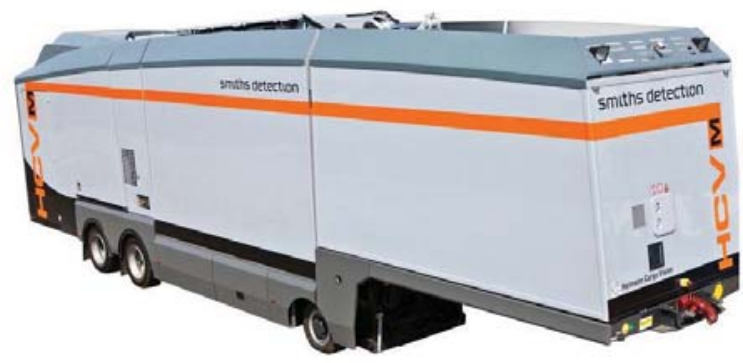

Figure 1. The HCVM-T towable X-ray system upgraded in C-BORD project.

\section{Rapidly relocatable tagged neutron inspection system (RRTNIS)}

The RRTNIS system is based on neutron generator with associated particles, allowing for choosing the coincidence with neutrons emitted in a specific direction in form of cone. Precursor of this device was primarily developed in EURITRACK project [4]. Similarly, the improved system will be used for searching tobacco, drugs and chemicals, performing the identification of chemical elements composition. The intention is to improve efficiency of the system and optimize the system weight in comparison with the EURITRACK project. Due to use of $14 \mathrm{MeV}$ neutrons and the fact that the space at border crossing or seaport is often limited, well optimized PE and Pb shielding were introduced.

The device design allows to dismantle in 48 hours and transport to another location after placing in a standard transportation container. The RRTNIS data analysis principle is presented in Figure 2.

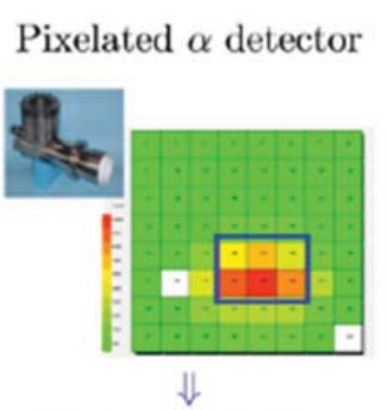

Neutron direction
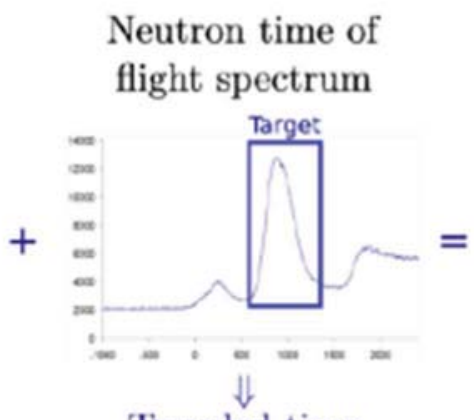

Traveled time

Distance traveled

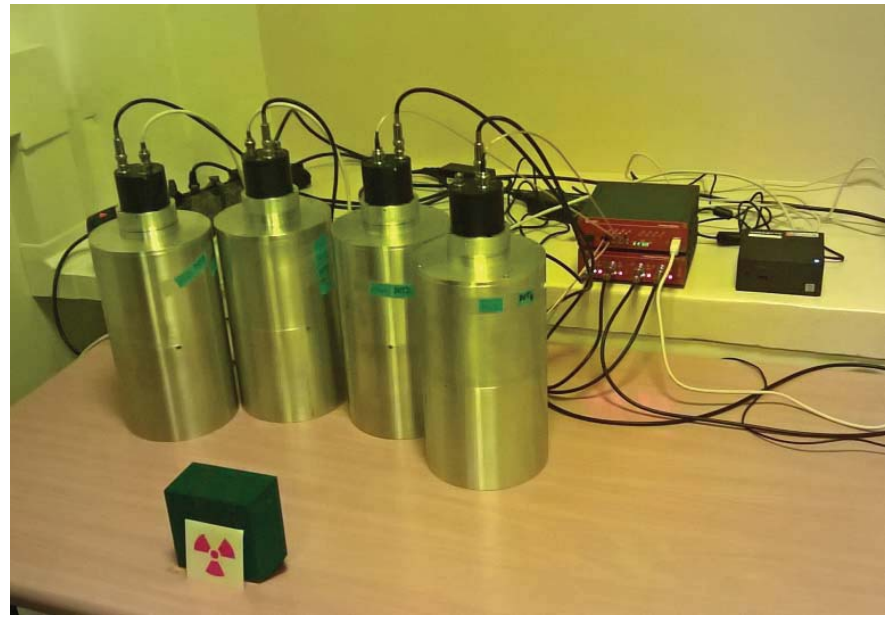

Figure 3. Fully operational detectors and data readout for photofission system. Detectors were designed at NCBJ, the electronics were produced by CAEN.

\section{Photofission system}

The photofission system for detection of special nuclear materials (SNM) and fertile materials, will be able to detect three main photofission signatures [5]:

- High energy delayed gamma-rays detection with use of large plastic scintillators [6], [7],

- Delayed neutrons [8]

- Prompt neutrons by means of Threshold Activation Detection (TAD) [9]-[12]

Moreover, a system for detection of early-delayed gamma rays is also developed, which will be useful for immediate classification of smuggled nuclear materials. For prompt neutron detection $\mathrm{CaF}_{2}$ detectors, designed at $\mathrm{NCBJ}$, was tested at CEA LIST SAPHIR facility and will be used during test at Rotterdam site. Detailed study of the $\mathrm{CaF}_{2}$ scintillator as the TAD detector was done in [13]. The fully operational

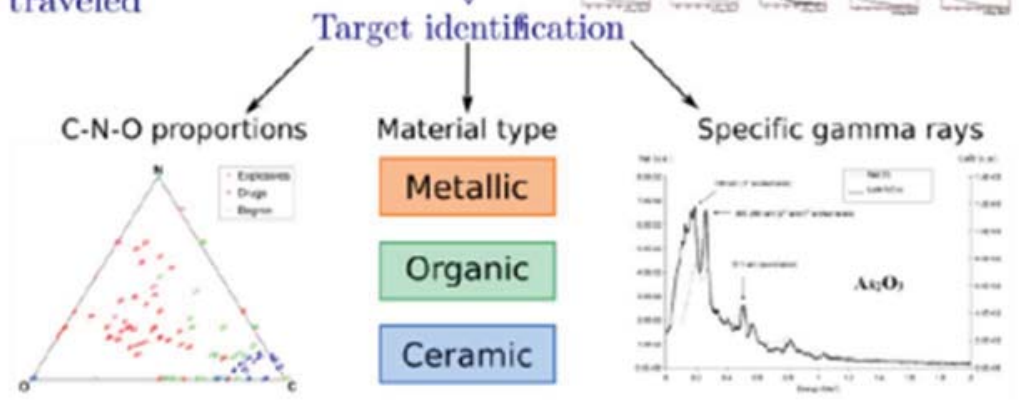

Figure 2. The RRTNIS data analysis principle. 
detectors with associated electronics from CAEN are shown in Figure 3. The detection is based on neutron activation through ${ }^{19} \mathrm{~F}(\mathrm{n}, \alpha){ }^{16} \mathrm{~N}$ reaction in the scintillator medium, resulting in emission of characteristic radiation - both gamma rays and beta particles - up to $10.4 \mathrm{MeV}$.

\section{E. Evaporation based detectors (SNIFFER)}

The evaporation based detection system [14], complementary to X-ray system, sucks vapor from the inspected container in order to find characteristic agents ascribed to tobacco, drugs or explosives or other illicit chemicals. The picture of a part of the analyzing device is presented in Figure 4.

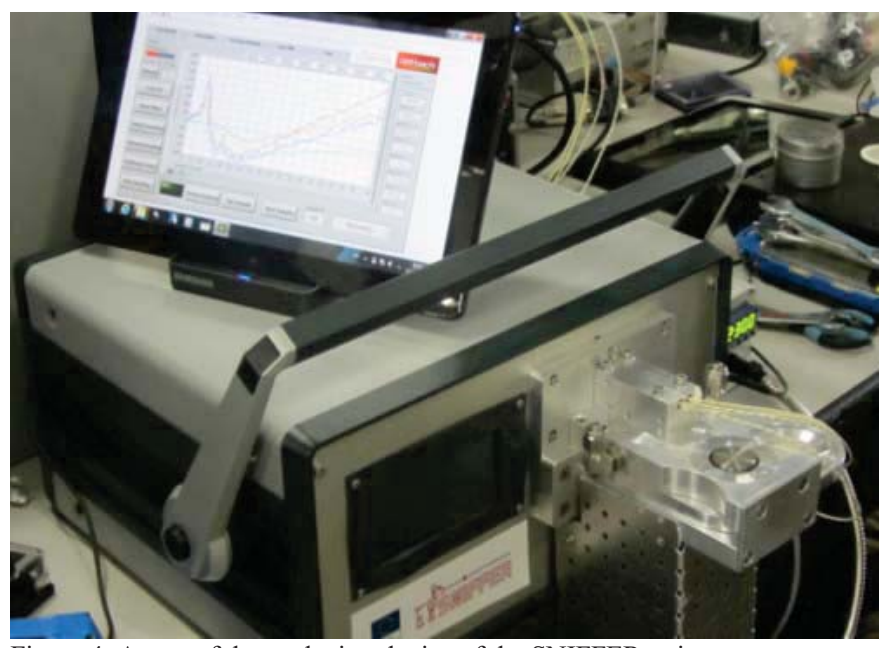

Figure 4. A part of the analyzing device of the SNIFFER unit.

\section{SUMMARY}

The document presents the overview of the systems developed in the C-BORD project. All these systems will be integrated into common software, providing information required by end-user from each system in a one frame. Finally, all devices will be tested at real conditions in a field trial at three border crossing - two seaports and one land border crossing. The main achievement of the C-BORD project is to provide complementary toolbox for high volume freight NII, resulting in better monitoring of goods transported to Europe in the effective and cost efficient way, and further, to increase the safety level in Europe.

\section{ACKNOWLEDGMENT}

This project has received funding from the European Union's Horizon 2020 research and innovation programme under grant agreement No 653323. Disclaimer: This text reflects only the author's views and the Commission is not liable for any use that may be made of the information contained therein.

\section{REFERENCES}

[1] "C-BORD Project - Effective Container Inspection at BORDer Control Points." [Online]. Available: http://www.cbord-h2020.eu/. [Accessed: 09-Nov-2017].

[2] "Symetrica LTD." [Online]. Available: http://www.symetrica.com/. [Accessed: 08-Nov-2017]

[3] Smiths Detection, "HCVM-T towable X-ray system.” 25-Apr-2017.
[4] B. Perot et al., "Development of the EURITRACK tagged neutron inspection system," Nucl. Instrum. Methods Phys. Res. Sect. B Beam Interact. Mater. At., vol. 261, no. 1-2, pp. 295-298, Sierpie 2007.

[5] T. Gozani, "Fission Signatures for Nuclear Material Detection," IEEE Trans. Nucl. Sci., vol. 56, no. 3, pp. 736-741, Jun. 2009.

[6] F. Carrel, M. Agelou, M. Gmar, and F. Lainé, "Detection of highenergy delayed gammas for nuclear waste packages characterization," Nucl. Instrum. Methods Phys. Res. Sect. Accel. Spectrometers Detect. Assoc. Equip., vol. 652, no. 1, pp. 137-139, Oct. 2011.

[7] P. Sibczynski et al., "Decay chains and photofission investigation based on nuclear spectroscopy of highly enriched uranium sample," Appl. Radiat. Isot., vol. 82, no. Supplement C, pp. 170-174, Dec. 2013.

[8] M. Gmar and others, "Assessment of actinide mass embedded in large concrete waste packages by photon interrogation and photofission," Appl. Radiat. Isot., vol. 63, no. 5-6, pp. 613-619, 2005.

[9] T. Gozani and others, "Neutron threshold activation detectors (TAD) for the detection of fissions," Nucl. Instrum. Methods Phys. Res. Sect. Accel. Spectrometers Detect. Assoc. Equip., vol. 652, no. 1, pp. 334 337, 2011.

[10] T. Gozani, "The investigation of fast neutron Threshold Activation Detectors (TAD)," in $2^{\text {nd }}$ Iinternational Workshop on Fast Neutron Detetctors and Applications, November 6-11, 2011, Ein Gedi, Israel, 2012.

[11] P. Sibczynski and others, "Verification of threshold activation detection (TAD) technique in prompt fission neutron detection using scintillators containing ${ }^{19} \mathrm{~F}, "$ JINST 010T0215, 2015.

[12] P. Sibczynski et al., "Comparison of prompt and delayed photofission neutron detection techniques using different types of radiation detectors," presented at the IEEE Nuclear Science Symposium / Medical Imaging Conference, Strasbourg, 2016.

[13] P. Sibczynski, "New perspectives for undoped CaF2 scintillator as a threshold activation neutron detector," Present. ANIMMA 2017 Conf.

[14] K. C. Persaud, P. Wareham, A. M. Pisanelli, and E. Scorsone, "Electronic Nose'-New Condition Monitoring Devices for Environmental Applications," Chem. Senses, vol. 30, no. 1, pp. 252253, Jan. 2005 